\title{
Roscovitine-treated HeLa cells finalize autophagy later than apoptosis by downregulating Bcl-2
}

\author{
AJDA COKER-GURKAN ${ }^{1}$, ELIF DAMLA ARISAN ${ }^{1}$, PINAR OBAKAN $^{1}$, PELIN OZFILIZ $^{1}$, \\ BETSI KOSE $^{1}$, GUVEN BICKICI ${ }^{1,2}$ and NARCIN PALAVAN-UNSAL ${ }^{1}$ \\ ${ }^{1}$ Department of Molecular Biology and Genetics, Istanbul Kultur University, Istanbul 34156, Turkey; \\ ${ }^{2}$ Department of Life and Sport Sciences, School of Science, University of Greenwich, Kent, UK
}

Received December 4, 2013; Accepted May 30, 2014

DOI: $10.3892 / \mathrm{mmr} .2014 .2902$

\begin{abstract}
The cell cycle is tightly regulated by the family of cyclin-dependent kinases (CDKs). CDKs act as regulatory factors on serine and threonine residues by phosphorylating their substrates and cyclins. CDK-targeting drugs have previously demonstrated promising effects as cancer therapeutics both in vitro and in vivo. Roscovitine, a purine-derivative and specific CDK inhibitor, has been demonstrated to arrest the cell cycle and induce apoptosis in a number of different cancer cell lines, including HeLa cervical cancer cells. In the present study, roscovitine was able to decrease both the cell viability and cell survival as well as induce apoptosis in a dose-dependent manner in HeLa cells by modulating the mitochondrial membrane potential. The decrease of anti-apoptotic B-cell lymphoma 2 (Bcl-2) and Bcl-2 extra large protein expression was accompanied by the increase in pro-apoptotic Bcl-2-associated X protein and P53-upregulated modulator of apoptosis expression. The marked decrease in Bcl-2 following exposure to roscovitine $(20 \mu \mathrm{M})$ for $48 \mathrm{~h}$ prompted us to determine the autophagic regulation. The outcome revealed that roscovitine triggered Beclin-1 downregulation and microtubule-associated light chain 3 cleavage starting from $12 \mathrm{~h}$ of incubation. Another biomarker of autophagy, p62, a crucial protein for autophagic vacuole formation, was diminished following $48 \mathrm{~h}$. In addition, monodansyl cadaverin staining of autophagosomes also confirmed the autophagic regulation
\end{abstract}

Correspondence to: Dr Elif Damla Arisan, Department of Molecular Biology and Genetics, Istanbul Kultur University, Atakoy Campus, 100 E-5 Highway, Istanbul 34156, Turkey

E-mail: d.arisan@iku.edu.tr

Abbreviations: $\quad$ ABTS, 2,2'-azino-di-3-ethylbenzthiazoline sulfonate 6 diammonium salt; AO, acridine orange; DiOC6, 3,3'-dihexyloxacarbocyanine iodide; DMEM, Dulbecco's modified Eagle's medium; DMSO, dimethylsulfoxide; MDC, monodansylcadaverine; PBS, phosphate-buffered saline; PI, propidium iodide; $\mathrm{POD}$, peroxidase

Key words: roscovitine, cervical cancer, autophagy, apoptosis, Bcl-2 by roscovitine treatment. The expression levels of different Bcl-2 family members determined whether apoptosis or autophagy were induced following incubation with roscovitine for different time periods. Downregulation of pro-apoptotic Bcl-2 family members indicated induction of apoptosis, while the downregulation of anti-apoptotic Bcl-2 family members rapidly induced autophagosome formation in HeLa cells.

\section{Introduction}

Cyclin-dependent kinases (CDKs) strictly orchestrate the cell cycle machinery through the binding to their specific cyclin counterparts at cell cycle checkpoints. The CDK-cyclin complex formation ensures the appropriate and accurate transition between cell cycle phases, which is essential for cell proliferation (1-3). Cancer cells frequently demonstrate de-regulated CDK activity that causes uncontrolled cell proliferation, which is a hallmark of malignant cell turnover. Therefore, recent approaches targeting CDKs with specific small molecule inhibitors have a crucial role both in developmental biology and cancer therapy. Roscovitine (CYC202 or seliciclib), a purine derivative, is a novel promising candidate for CDK inhibition and is able to induce apoptosis in a variety of cancer cell types; including lung, prostate, breast and colon cancers as well as multiple myeloma (4-5). Roscovitine mainly acts as a competitive inhibitor of CDKs while showing a broad affinity for specific CDK ends, and blocks the ATP-binding catalytic site of kinases $(1,3)$. Recent studies demonstrated that roscovitine induces cell cycle arrest at the G1/S or G2/M phase transition in MCF-7 breast (1), lung cancer (2), HeLa cervical (3) and HCT116 colon cancer (4) cells. In addition, roscovitine treatment may synergize the anti-tumor effects of other chemotherapeutics by inducing further apoptotic cell death (5).

Although previous studies have established the apoptotic potential of roscovitine in HeLa cells (3), the autophagic regulation following roscovitine treatment has not been fully elucidated. Autophagy is a regulated process of degradation and recycling of cellular components in vacuoles inside the cell, allowing organelle and energy turnover. Autophagy has a crucial role in tissue dynamics, homeostasis, development and disorders. Autophagy is responsible for the degradation of cytoplasmic macromolecular and protein structures 
and damaged or aged organelles in the cells. The most significant marker of autophagy is the appearance of double membrane-enclosed vesicles, which engulf portions of cytoplasm and/or organelles in the cytoplasm (6-8). Due to different stress factors, including starvation, autophagy can result in cellular adaptation as an alternative to cell death or survival. Although autophagy has been considered to be a stress adaptation mechanism in response to certain conditions, including starvation, apoptosis and autophagy is now considered to have a specific function. In variable cellular settings and in the presence of different external factors, autophagy is regarded as an alternative cell death pathway. Depending on the type of the stimulus, the two types of cell death may be triggered at the same time, corresponding to each other by common upstream signals, or occasionally the cell switches in between these death pathways in a successive manner. Thus, the clarification of the joint molecular targets in both apoptosis and autophagy regulation processes is critical to evaluate the therapeutic potential of novel chemotherapeutic drugs. One of the links between apoptosis and autophagy is B-cell lymphoma 2 (Bcl-2), which is an anti-apoptotic member of the Bcl-2 family. Bcl-2 exerts a dual role to inhibit apoptosis and eliminate pro-apoptotic Bcl-2 family members or autophagy by binding Beclin-1, which has a similar structure to that of pro-apoptotic $\mathrm{Bcl}-2$ proteins. Beclin-1 is a Bcl-2 homology (BH) 3 only protein which binds to anti-apoptotic Bcl-2 family members; myeloid cell leukemia-1 (Mcl-1), Bcl-2 or Bcl-2 extra large $\left(\mathrm{Bcl}_{\mathrm{L}}\right)(9,10)$. It has been suggested that anti-apoptotic Bcl-2 family members exert a dual role in inhibiting both apoptotic and autophagic pathways in the cell. Beclin-1 is an initiator factor for autophagy and recruits Vps34 and Vps15 to form pre-autophagosome structures (11). Therefore, the expression profile of Beclin-1 akin to Bcl-2 may be a crucial switch molecule between apoptosis and autophagy (12).

The present study aimed to examine the potential therapeutic role of roscovitine in HeLa cervical cancer cells by investigating apoptotic and autophagic molecular signaling molecules in a time-dependent manner.

\section{Materials and methods}

Drugs, chemicals and antibodies. Roscovitine (seliciclib, 2-(1ethyl-2-hydroxy-ethylamino)-6-ben-

zylamino-9-isopropylpurine)

was purchased from Sigma-Aldrich (St Louis, MO, USA) and dissolved in dimethyl sulfoxide (DMSO) to establish a $10 \mathrm{mM}$ stock solution, and the aliquots were maintained at $-20^{\circ} \mathrm{C}$. Propidium iodide (PI), DAPI, 3,3'-dihexyloxacarbocyanine iodide (DiOC6) and acridine orange (AO) were purchased from Applichem (Darmstadt, Germany). Monodansylacadaverine (MDC) was purchased from Sigma-Aldrich. The monoclonal anti-rabbit antibodies against Bcl-2-associated X (Bax; 1:1,000), Bcl-2 (1:1,000), Bcl- $\mathrm{x}_{\mathrm{L}}$ (1:1,000), P53-upregulated modulator of apoptosis (Puma; 1:1,000), Beclin-1 (1:1,000), microtubule-associated light chain 3B (LC3B; 1:1,000), autophagy protein (Atg)5 (1:1,000), Atg12 (1:1,000), p62 (1:1,000), $\beta$-actin (1:1000) and horseradish peroxidase (HRP)-conjugated secondary anti-rabbit $(1: 5,000)$ were purchased from Cell Signaling Technology, Inc. (Danvers, MA, USA).
Cell culture. HeLa (CCL-2) cervical adenocarcinoma cells were purchased from the American Type Culture Collection (Manassas, VA, USA). The cells were maintained in high-glucose Dulbecco's modified Eagle's medium (DMEM; PAN Biotech, Aidenbach, Germany) with $10 \%$ fetal calf serum (FCS; PAN Biotech) and $100 \mathrm{U} / 100 \mathrm{mg} \mathrm{ml}^{-1}$ penicillin/streptomycin (PAN Biotech) at $37^{\circ} \mathrm{C}$ in a humidified $5 \% \mathrm{CO}_{2}$ incubator (Hera Cell 150i; Thermo Fisher Scientific, San Jose, CA, USA). Serum starvation of cells was performed via incubation of cells with DMEM and $0.5 \%$ FCS for $2 \mathrm{~h}$ prior to staining with MDC.

MTT cell viability assay. To determine the cytotoxicity of roscovitine treatment, an MTT cell viability assay [Sigma; $50 \mathrm{mg} / \mathrm{ml}$ in phosphate-buffered saline (PBS)] was used. For this purpose, $1 \times 10^{4}$ cells were seeded in each well of a 96-well plate and treated with roscovitine for $24 \mathrm{~h}$. MTT was added and the cells were incubated at $37^{\circ} \mathrm{C}$ for $4 \mathrm{~h}$. Following removal of the MTT reagent including the media, $100 \mu \mathrm{l}$ DMSO was added to each well and cells were incubated for $5 \mathrm{~min}$ in the dark. Absorbance was determined at $570 \mathrm{~nm}$ (A570) using a microplate reader (Model 680; Bio-Rad Laboratories, Inc., Hercules, CA, USA).

Survival assay (trypan blue dye exclusion assay). A total of $5 \times 10^{4}$ cells per well were seeded into six-well plates and treated with roscovitine for 24,48 and $72 \mathrm{~h}$. The cells were then counted by staining with $0.4 \%$ (w/v) trypan blue dye. Viable and dead cells were counted under light microscopy (IX71; Olympus Corporation, Tokyo, Japan). The number of viable cells (y-axis) was plotted against the time (x-axis).

Apoptotic cell death ELISA assay. The ability of roscovitine to induce apoptosis was determined by the Cell Death Detection ELISA PLUS kit (Roche, Indianapolis, IN, USA). The cell lysates were placed in a streptavidin-coated microplate. A mixture of anti-histone-biotin and anti-DNA-peroxisidase (POD) was incubated for $2 \mathrm{~h}$ at $15-25^{\circ} \mathrm{C}$. Following removal of unbound antibodies by washing, POD was determined colorometrically at $405 \mathrm{~nm}$ with 2,2'-azino-di-(3-ethylbenzthiazoline sulfonate (6) diammonium salt (ABTS), which was used as a substrate.

Fluorescence microscopy. The HeLa cells were seeded at a density of $5 \times 10^{4}$ per well in 12 -well plates and treated with $20 \mu \mathrm{M}$ roscovitine for 24 and $48 \mathrm{~h}$, respectively. The cells were stained with different fluorescent dyes which are summarized in Table I. Following the indicated incubation period of the cells at $37^{\circ} \mathrm{C}$, the dye including the media was removed, $1 \mathrm{X}$ PBS was added and cells were visualized by fluorescent microscopy according to their specific excitation and emission wavelengths at the indicated time-points.

Immunoblot analysis. The cells were lysed on ice using ProteoJET mammalian cell lysis reagent (Fermentas, Glen Burnie, MD, USA) containing protease inhibitor (Roche, Mannheim, Germany). The cell lysates were centrifuged at $16,100 \mathrm{x} \mathrm{g}$ for $20 \mathrm{~min}$ at $4^{\circ} \mathrm{C}$ and the protein concentration was measured by a Bradford Assay (Bio-Rad Laboratories, Inc.). Following separation of denatured proteins according to size by SDS-PAGE, the proteins were transferred onto a polyvinylidene 
Table I. Fluorescence dyes to detect apoptosis and autophagy.

\begin{tabular}{lcccc}
\hline Dye & Concentration & Incubation time $(\mathrm{min})$ & Excitation $(\mathrm{nm})$ & Emission $(\mathrm{nm})$ \\
\hline PI & $5 \mathrm{mg} / \mathrm{ml}$ & 30 & 536 & 617 \\
DiOC6 & $4 \mathrm{nM}$ & 15 & 488 & 525 \\
DAPI & $5 \mathrm{mg} / \mathrm{ml}$ & 10 & 350 & 570 \\
AO & $5 \mathrm{mg} / \mathrm{ml}$ & 10 & 460 & 650 \\
MDC & $10 \mathrm{mg} / \mathrm{ml}$ & 30 & 510 & 595 \\
\hline
\end{tabular}

PI, propidium iodide; DiOC6, 3,3'-dihexyloxacarbocyanine iodide, AO, acridine orange; MDC, monodansylcadaverine.
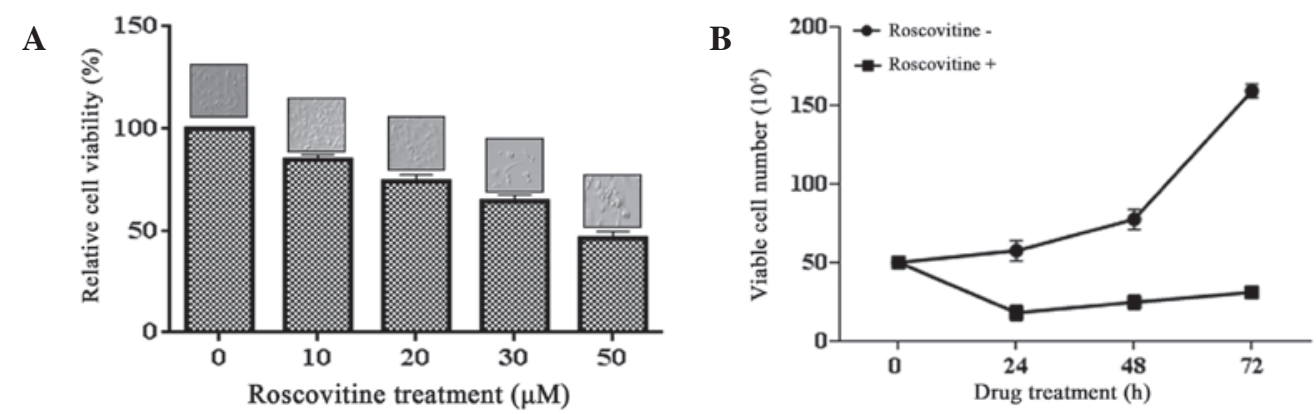

Figure 1. Roscovitine decreases cell viability and inhibits proliferation in a dose-dependent manner in HeLa cells. (A) Roscovitine decreases the cell viability in a dose-dependent manner. The HeLa cells were seeded at a density of $1 \times 10^{4}$ cells/well and treated with various concentrations of roscovitine (0-50 $\left.\mu \mathrm{M}\right)$ for $24 \mathrm{~h}$. The bar histograms represent the mean $\pm \mathrm{SD}$ of four independent experiments with at least four replicates $(\mathrm{P}<0.0001$, vs the untreated control group). The representative morphological observations are displayed in the bar chart. (B) Roscovitine treatment suppressed the proliferation rate of HeLa cells within $24 \mathrm{~h}$. The cells were seeded at a density of $1 \times 10^{5} /$ well in six-well plates and treated with $20 \mu \mathrm{M}$ roscovitine for 24,48 and $72 \mathrm{~h}$. At these time-points, the cells were counted each day following staining with trypan blue. The lines in the graph show the number of unstained viable cells presented as the mean \pm SD of three independent experiments with at least four replicates ( $\mathrm{P}<0.0001$, vs the untreated control group). SD, standard deviation.

fluoride membrane (Thermo Scientific) where they were labeled using antibodies specific to the target protein. The gels obtained by SDS-PAGE were placed in the Trans-Blot Turbo transfer system (Bio-Rad Laboratories) and protein transfer was conducted at $25 \mathrm{~V}$ for $5 \mathrm{~min}$. The membrane was blocked in $5 \%$ bovine serum albumin/TBS containing $0.1 \%$ Tween-20 (TBST) for $2 \mathrm{~h}$ at room temperature, and then incubated with primary antibodies for each target; Bax, Puma, Bcl-2, Beclin-1, LC3B, Atg5, Atg12, p62 and $\beta$-actin overnight at $4^{\circ} \mathrm{C}$ and further incubated with anti-rabbit immunoglobulin G-HRP secondary antibody overnight at $4^{\circ} \mathrm{C}$. Following washing with TBST, the proteins were detected using Super Signal West FemtoLuminol/Enhancer Solution (Thermo Scientific) and exposedtoHyperfilm-enhancedchemiluminescence(Amersham Pharmacia Biotech, Piscataway, NJ, USA). Densitometric analysis of immunoblots was performed using Image $\mathbf{J}$ (National Institutes of Health, Bethesda, MD, USA) and GraphPad Prism 6 (GraphPad Software, Inc., La Jolla, CA, USA) software; and all of the proteins were quantified relative to the loading control.

Determination of mitochondrial membrane potential assay. The HeLa cells $\left(1 \times 10^{5}\right)$ were seeded in 12 -well plates, treated with the indicated concentration of roscovitne and allowed to attach overnight. The cells were washed once with 1X PBS, and stained with $0.4 \mathrm{mM} \mathrm{DiOC}_{6}$ (Molecular Probes Life Technologies, Carlsbad, CA, USA) fluorescent probe. Mitochondrial membrane potential (MMP) loss was measured using a Fluoroskan Ascent fluorometer (excitation/emission $=488 \mathrm{~nm} / 525 \mathrm{~nm}$; Thermo Labsystems, Milford, MA, USA).

Statistical analysis. All of the experiments were statistically analyzed using GraphPad Prism 6 software. Error bars in the graphs were generated using \pm standard deviation values. A $\mathrm{P}<0.05$ was considered to indicate a statistically significant difference.

\section{Results}

Roscovitine decreases cell viability and induces apoptosis in a dose-dependent manner. The effect of roscovitine on the viability of HeLa cells was investigated by an MTT assay. Exposure of the cells to roscovitine (0-50 $\mu \mathrm{M})$ for $24 \mathrm{~h}$ decreased the cell viability in a dose-dependent manner $(\mathrm{P}<0.0001$, each compared with the untreated control). A moderate cytotoxic effect was triggered following $20 \mu \mathrm{M}$ roscovitine treatment, which caused $25 \%$ cell viability loss compared with the untreated control samples. The highest concentration of $50 \mu \mathrm{M}$ roscovitine reduced the cell viability ratio by $50 \%$ within $24 \mathrm{~h}$ (Fig. 1A).

To confirm the cytotoxic effect of roscovitine on cell proliferation in HeLa cells, a trypan blue exclusion assay was also performed (Fig. 1B). Exponentially growing HeLa cells were treated with roscovitine $(20 \mu \mathrm{M})$ for different time periods within $72 \mathrm{~h}$. The doubling time of HeLa cells was determined 


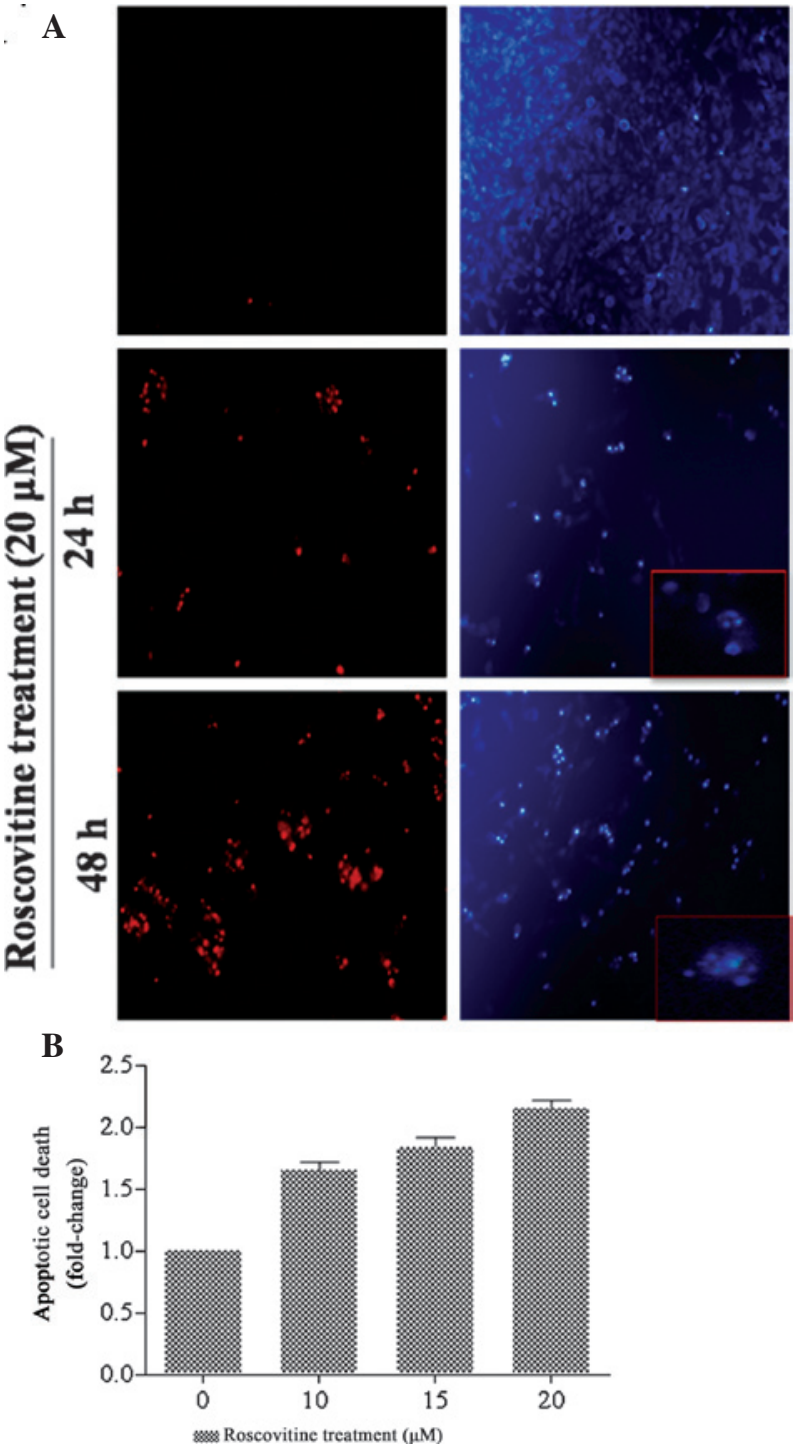

Figure 2. Roscovitine-induced apoptosis. (A) Morphological changes due to roscovitine-induced apoptosis were determined by fluorescent microscopy. The effect of roscovitine on apoptosis was observed via propidium iodide and 6-diamidino-2-phenylindole staining. Representative images were taken at magnification of $\times 100-400$. (B) Cells were seeded at a density of $1 \times 10^{4}$ in 96-well plates and treated with various concentreations of roscovitine $(0-20 \mu \mathrm{M})$ for $24 \mathrm{~h}$. Apoptotic cell death was evaluated by the Cell Death ELISA Plus assay $(\mathrm{P}<0.0001$ vs. the untreated control sample). For all of the measurements, the error bars represent the mean \pm standard deviation of four independent experiments with at least four replicates.

to be $23 \mathrm{~h}$, which was in accordance with the information provided by the supplier. Treatment of the cells with roscovitine suppressed the cell viability following $24 \mathrm{~h}$. However, the cells gained proliferation capacity following long-term exposure to roscovitine for 48 and $72 \mathrm{~h}$. Although roscovitine diminished the cell proliferation within $24 \mathrm{~h}$, it was concluded that longer treatment with roscovitine may lead to the accumulation of resistance factors in HeLa cells $(\mathrm{P}<0.0001)$.

Following exposure of HeLa cells to roscovitine for 24 and $48 \mathrm{~h}$, the cells were stained with PI and DAPI to identify the cell death mechanism (Fig. 2A). The percentage of PI-positive cells, which are regarded as dead cells, was increased following roscovitine treatment of HeLa cells (red stain) compared with the untreated cells in a time-dependent manner. In addition,
A

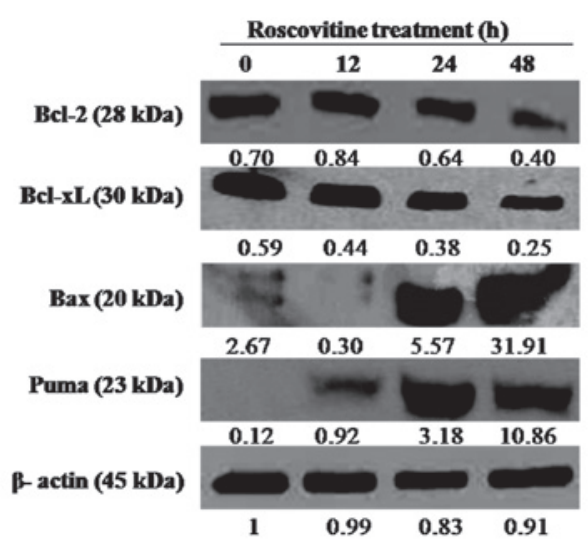

B Roscovitine treatment $(20 \mu \mathrm{M})$

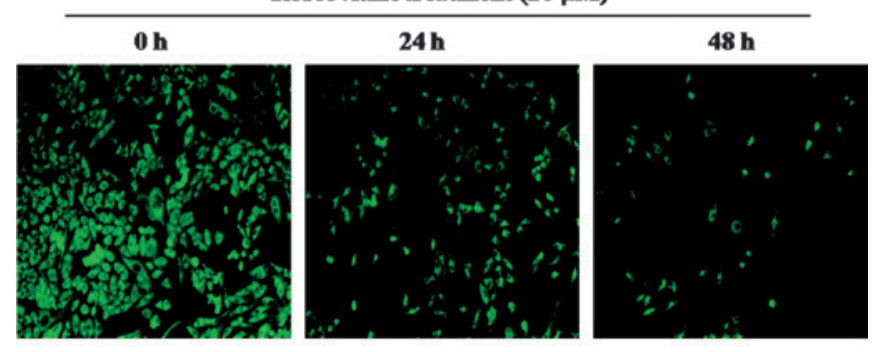

C

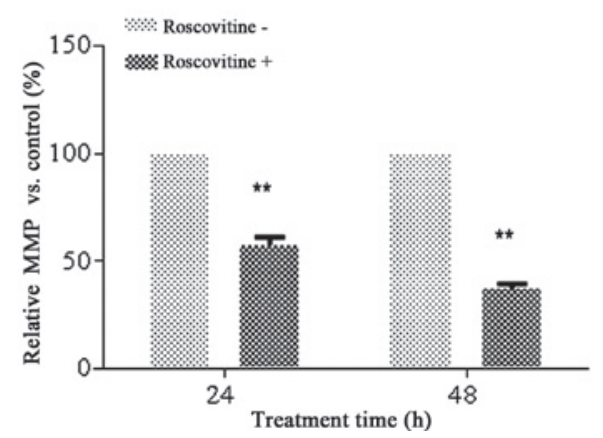

Figure 3. Roscovitine induced mitochondria-mediated apoptosis. (A) HeLa cells were seeded at a density of $1 \times 10^{6}$ in $60-\mathrm{mm}$ plates and treated with roscovitine $(20 \mu \mathrm{M})$ for 12,24 and $48 \mathrm{~h}$. A total of $20 \mu \mathrm{g}$ of protein lysate was purified by $12 \%$ SDS-PAGE. $\beta$-actin was used as a loading control. (B) The loss of MMP was determined by fluoresence microscopy (magnification, $\mathrm{x} 200)$. (C) The MMP was measured by a fluorometer $(\mathrm{P}<0.0001$, vs the untreated control group; excitation, $485 \mathrm{~nm}$; emission, $538 \mathrm{~nm}$ ). MMP, mitochondrial membrane potential; Bcl-2, B-cell lymphoma 2; Bcl-xL, Bcl-2 extra large; Bax, Bcl-2-associated X; PUMA, P35-upregulated modulator of apoptosis.

DAPI staining was performed to identify apoptotic cells which exhibited fragmented DNA and nuclear condensation. The number of apoptotic cells (bright blue stain) was increased following 24 and $48 \mathrm{~h}$ roscovitine treatment compared with the untreated control cells. According to the fluorescence images, the ratio of nuclear condensation was increased following $48 \mathrm{~h}$ of roscovitine treatment.

In order to investigate the cytotoxic effect of roscovitine on HeLa cells, a Cell Death ELISA assay was performed, which demonstrated the cleavage of DNA due to drug treatment (Fig. 2B). Exposure of cells to lower concentrations of roscovitine $(10$ and $15 \mu \mathrm{M})$ induced apoptotic cell death by 1.65 and 1.85-fold compared with untreated control samples, respectively. The appropriate concentration of roscovitine, $20 \mu \mathrm{M}$, 
A

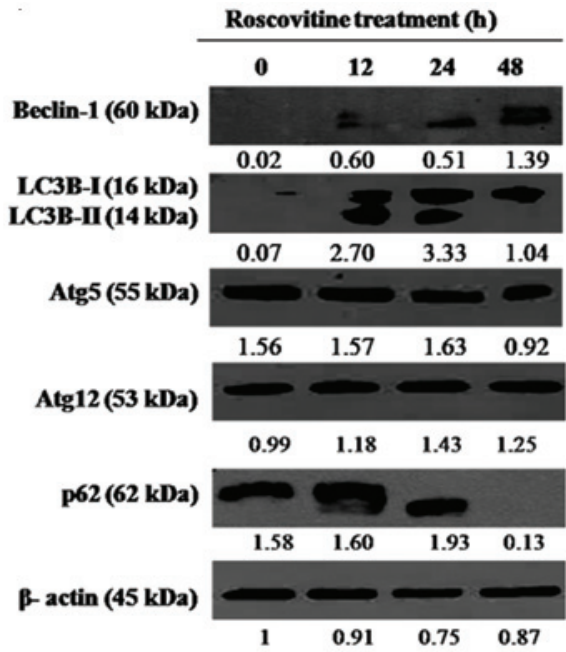

B

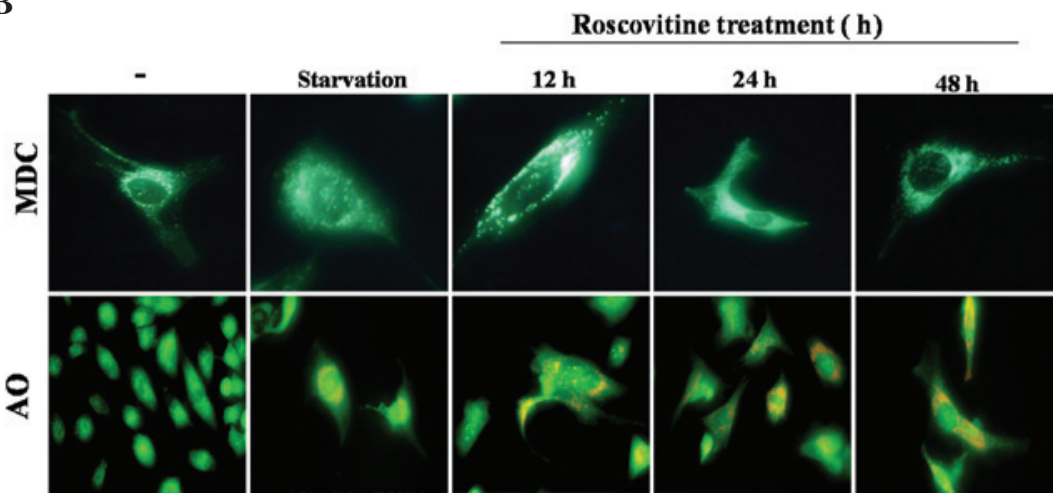

Figure 4. Roscovitine induced autophagy. (A) HeLa cells were seeded at a density of $1 \times 10^{6}$ in $60 \mathrm{~mm}$ plates and treated with roscovitine $(20 \mu \mathrm{M})$ for 12,24 and $48 \mathrm{~h}$. A total of $20 \mu \mathrm{g}$ of protein lysate was purified by $12 \%$ SDS-PAGE. $\beta$-actin was used as a loading control. (B) The effect of roscovitine on autophagic vesicles was determined by AO and MDC staining. Representative images were taken at a magnification of x100-400. AO, acridine orange; MDC, monodansylcadaverine.

increased apoptosis by 2 -fold compared with the untreated control cells $(\mathrm{P}<0.0001$, each compared with the untreated control). Therefore, it was concluded that roscovitine treatment for $24 \mathrm{~h}$ decreased the cell viability and induced apoptosis in a dose-dependent manner.

Roscovitine induces apoptosis and autophagy through modulating levels of Bcl-2 family members. Since Bcl-2 family members have a decisive role in apoptotic cell death mechanisms, the effect of roscovitine on the expression levels of pro- and anti-apoptotic Bcl-2 family member proteins in HeLa cells was determined. As revealed in Fig. 3A, roscovitine treatment decreased the $\mathrm{Bcl}-2$ and $\mathrm{Bcl}-\mathrm{x}_{\mathrm{L}}$ expression levels time-dependently, whereas the activation of pro-apoptotic Bcl-2 family members, Puma and Bax, was observed as an early response. Although roscovitine treatment for $24 \mathrm{~h}$ downregulated $\mathrm{Bcl}-\mathrm{x}_{\mathrm{L}}$ expression levels, no significant alteration was observed for Bcl-2 at the same time-point. It was concluded that roscovitine is an apoptosis-inducing agent via modulating the ratio between anti and pro-apoptotic Bcl-2 family members in HeLa cells.

In order to examine the potential role of roscovitine on mitochondria-mediated apoptosis, DiOC6 staining was performed (Fig. 3B and C). The number of viable cells (green stained), which have normal mitochondrial function, was gradually decreased in a time-dependent manner. The loss in mitochondrial membrane potential (MMP) was also confirmed by fluorometric analysis (excitation, $485 \mathrm{~nm}$; emission, $538 \mathrm{~nm}$ ).

To investigate the effect of roscovitine on autophagic regulation mechanisms, the expression levels of the key signaling molecules associated with autophagy were investigated following roscovitine treatment in HeLa cells. As demonstrated in Fig. 4A, Beclin-1 expression levels were progressively upregulated with increasing exposure time to the drug in HeLa cells. Treatment with roscovitine for 12 and $24 \mathrm{~h}$ upregulated the expression of two LC3 isoforms (LC3-I and LC3-II); however, long-term treatment for $48 \mathrm{~h}$ with the drug downregulated the LC3-II isoform in HeLa cells (Fig. 4A). By contrast, the expression profiles of Atg5 and Atg12 were not altered following roscovitine treatment. p62 is referred to as a significant biomarker for the autophagic vacuole formation process. In the present study, it was determined that roscovitine treatment at $48 \mathrm{~h}$ eliminated the p62 expression levels in $\mathrm{HeLa}$ cells. Therefore, it was concluded that autophagic mechanisms are finalized later than roscovitine-induced apoptosis.

To demonstrate the stages of roscovitine-induced autophagy, cells were stained with MDC or AO (Fig. 4B). MDC staining was performed to visualize acidic autophagic vesicles. As a positive control, nutrient-deprived cells were stained with MDC, displaying bright green dots. Although MDC-stained cells were identified following 12, 24 and $48 \mathrm{~h}$ of roscovitine treatment, maximum fluorescent staining was observed after $12 \mathrm{~h}$. The lysosomotrophic dye AO was used 
to identify lysosomes. As a positive control, nutrient-deprived cells were stained with $\mathrm{AO}$, displaying bright red dots. The accumulation of $\mathrm{AO}$ dye was increased following roscovitine treatment in a time-dependent manner.

\section{Discussion}

Cervical cancer is the second most common cancer type among females worldwide and the disease etiology is mostly linked to infection with Human Papilloma Virus (HPV) $(13,14)$. Depending on the tumor size the preferred treatments for cervical cancer are medical surgery, radiation therapy, chemotherapy or their combination. However, the presence of an increasing number of recurrent cases demonstrates an urgent requirement for improvement of therapeutic application models. Although a number of the classical chemotherapeutic agents, including cisplatin, mitomycin $\mathrm{C}$ and bleomycin, are used as therapeutic agents against cervical cancer, these drugs induce intrinsic resistance factors, which renders the efficiency of therapy $(15,16)$. Therefore, the development of novel anti-tumor agents is critical for the treatment of cervical cancer patients.

Since the loss of functional cell cycle control is a common problem in numerous malignancies, CDK inhibitors are considered to inhibit the uncontrolled proliferation in cancer cells. Roscovitine was the first orally bioavailable CDK inhibitor to enter clinical trials for breast, lung, B-cell lymphoma and advanced solid tumors (17-19). Previous studies established that roscovitine predominantly inhibits CDK2 and induces cell cycle arrest at G2/M phase. In the present study, it was identified that roscovitine decreases cell viability in a dose-dependent manner in HeLa cells. A moderately cytotoxic concentration of the drug $(20 \mu \mathrm{M})$ prevented cell proliferation and induced apoptosis by 2 -fold compared with the untreated control cells within $24 \mathrm{~h}$. As demonstrated in previous studies, roscovitine induced apoptosis mainly through modulating MMP and activating caspase- $9,-3$ and poly(adenosine diphosphate-ribose) polymerase cleavage in different cancer cells $(4,18,20-28)$. Similar to these observations, it was determined in the present study that $20 \mu \mathrm{M}$ roscovitine decreased the MMP in a time-dependent manner in HeLa cells [MMP loss, 45\% (24 h) and 65\% (48 h) vs. the control group, respectively]. Although roscovitine $(20 \mu \mathrm{M})$ upregulated Bax and Puma expression levels time-dependently, anti-apoptotic $\mathrm{Bcl}-2$ was downregulated following long-term exposure of cells to the drug for $48 \mathrm{~h}$. By contrast, $\mathrm{Bcl}-\mathrm{x}_{\mathrm{L}}$ was downregulated in a time-dependent manner following roscovitine treatment. Therefore, it was concluded that roscovitine exerts its apoptotic effect on HeLa cells by altering the MMP and modulating Bcl-2 family members in HeLa cells. A previous study revealed that roscovitine $(20 \mu \mathrm{M})$ induced apoptosis via reducing the cell proliferation caused by $\mathrm{G} 2 / \mathrm{M}$ cell cycle arrest in tamoxifen therapy-resistant breast cancer cell lines. In addition, roscovitine successfully reduced breast tumor volume, which was evaluated in an in vivo xenograft tumor model (26). Although roscovitine mostly activated caspases to induce the apoptotic cell death mechanism (4), it was established that roscovitine may successfully induce apoptosis in caspase-3 null MCF-7 cells through upregulating the p53 signaling pathway (29). In response to this finding, the present study aimed to examine the potential role of roscovitine on autophagy, which is also accepted as a caspase-independent cell death type in HeLa cells. Previously, it was demonstrated that roscovitine treatment enhanced doxorubicin-induced apoptosis as well as autophagy in sarcoma cell line models (30). In addition, roscovitine downregulated mitogen-activated protein kinase $1 / 3$, which exhibits a high activity in mature oocytes of mammals, frogs or invertebrates, such as starfish, to maintain cell cycle arrest. By this way, mature oocytes underwent apoptosis if they were not fertilized. Roscovitine increased calcium-dependent mitotic cell entry and delayed cell death in sea urchins by downregulating MAPK1/3. Based on these observations, it was suggested that autophagy with a survival-promoting function may be activated when cell cycle arrest was triggered by a specific CDK inhibitor (31). The molecular targets of roscovitine on the autophagic regulation processes have not been fully elucidated yet. To examine its possible molecular targets, the present study first conducted experiments to clarify the potential effect of roscovitine on the key converging molecules between apoptosis and autophagy. Beclin-1 is an important mediator between cell death and survival mechanisms due to its binding ability to anti-apoptotic Bcl-2 family members. Pro-apoptotic Bcl-2 family members have a crucial role in the dissociation of the complex of Beclin-1 and Bcl-2. It was suggested that upregulation of several pro-apoptotic $\mathrm{Bcl}-2$ family members, including Bim $_{\mathrm{EL}}$, Bcl-2-interacting killer, Bcl-2 homology domain 3 interacting-domain death agonist, Bcl-2-associated death promoter, Puma, but not Bax or Bak, competitively bind to hydrophobic grooves on Bcl-2 and lead to autophagy by inducing the dissociation of Beclin-1 (32). Beclin-1 homodimerization due to $\mathrm{Bcl}-2$ interaction renders autophagic regulation in the cells by preventing heterodimerization of Beclin-1 with Atg14 or other complex proteins. In addition, the interaction between $\mathrm{Bcl}-2$ and Beclin-1 is regulated by c-Jun N-terminal kinase and extracellular signal-regulated kinase (ERK) led to phosphorylation of Bcl-2 or Beclin-1, respectively (33). Death-associated protein kinase is one of the crucial targets of ERK, which consequently induces the phosphorylation of Beclin-1 (34). It was identified that, although HeLa cells were lacking basal Beclin-1 expression, roscovitine treatment for $48 \mathrm{~h}$ upregulated the expression levels of Beclin-1. Concomitantly, Bcl-2 downregulation due to roscovitine treatment was marked. It was concluded that the upregulation of pro-apoptotic Puma and Bax was noteworthy following drug treatment for up to $48 \mathrm{~h}$, which may dissociate the Beclin-1 and Bcl-2 complex and lead to heterodimerization of autophagy complex proteins. Additionally, LC3, a cytosolic soluble protein, was cleaved during autophagic induction and its involvement in the autophagic vacuole membrane formation was determined. The 18 and $16 \mathrm{kDa}$ forms of LC3 were called LC3-I and LC3-II, respectively. Amino acid starvation-induced increases in the levels of LC3-II were found to be associated with membrane compartments, whereas LC3-I was localized in the cytoplasm (35). Previous findings stated that roscovitine may synergize both apoptosis and autophagy by enhancing the DNA-damaging effect of doxorubicin in different sarcoma cell lines (30). The number of green fluorescence protein-tagged LC3 puncta was increased due to prolonged cell cycle arrest at G2/M phase triggered by roscovitine treatment in the presence of doxorubicin, a DNA damage-inducing agent. AO staining, which was concentrated in acidic vacuoles, also confirmed that roscovitine synergized the doxorubicin-induced autopha- 
gosome formation. In addition to Beclin-1 upregulation, LC3 cleavage was increased following roscovitine treatment of $\mathrm{HeLa}$ cells for $12 \mathrm{~h}$. This suggested that when pro-apoptotic Bcl-2 family members were upregulated in a parallel manner, the autophagy-inducing signaling molecules were also upregulated. p62 has also been demonstrated to be an autophagic marker, which is depleted when autophagosome complexes are engulfed with lysosomes. Exposure of HeLa cells to roscovitine for $48 \mathrm{~h}$ eliminated the p62 expression levels. Therefore, lysosomes induced the degradation of p62 and LC3-II. To confirm lysosome-mediated degradation of p62 and LC3-II (16 kDa) in HeLa cells, AO staining was performed. The perceptible acidic vacuoles were distinguished gradually in a time-dependent manner following the exposure of cells to roscovitine. By contrast, as an early response to roscovitine treatment at $12 \mathrm{~h}$, MDC staining indicated acidic vacuole formation in HeLa cells. Therefore, it was concluded that roscovitine $(20 \mu \mathrm{M})$ induced both apoptosis and autophagy, with each process following one another in a time-dependent manner.

\section{Acknowledgements}

The authors are grateful to the Istanbul Kultur University Scientific Projects Support Center.

\section{References}

1. Wesierska-Gadek J, Gueorguieva M, Wojciechowski J and Horky M: Cell cycle arrest induced in human breast cancer cells by cyclin-dependent kinase inhibitors: a comparison of the effects exerted by roscovitine and olomoucine. Pol J Pharmacol 56: 635-641, 2004

2. Zhang T, Jiang T, Zhang F, et al: Involvement of p21Waf1/Cip1 cleavage during roscovitine-induced apoptosis in non-small cell lung cancer cells. Oncol Rep 23: 239-245,

3. Wesierska-Gadek J, Wandl S, Kramer MP, et al: roscovitine up-regulates p53 protein and induces apoptosis in human $\mathrm{HeLaS}(3)$ cervix carcinoma cells. J Cell Biochem 105: 1161-1171, 2008.

4. Arisan ED, Coker A and Palavan-Ünsal N: Polyamine depletion enhances the roscovitine-induced apoptosis through the activation of mitochondria in HCT116 colon carcinoma cells. Amino Acids 42, 655-665, 2012.

5. Appleyard MV, O'Neill MA, Murray KE, et al: Seliciclib (CYC202, R-roscovitine) enhances the antitumor effect of doxorubicin in vivo in a breast cancer xenograft model. Int J Cancer 124: 465-472, 2009.

6. Kondo Y, Kanzawa T, Sawaya R and Kondo S: The role of autophagy in cancer development and response to therapy. Nat Rev Cancer 5: 726-734, 2005.

7. Gozuacik D and Kimchi A: Autophagy and cell death. Curr Top Dev Biol 78: 217-245, 2007.

8. Maiuri MC, Zalckvar E, Kimchi A and Kroemer G: Self-eating and self-killing: crosstalk between autophagy and apoptosis. Nat Rev Mol Cell Biol 8: 741-752, 2007.

9. Wang J: Beclin 1 bridges autophagy, apoptosis and differentiation. Autophagy 4: 947-948, 2008.

10. $\mathrm{Ku} \mathrm{B}$, Woo JS, Liang C, et al: An insight into the mechanistic role of Beclin 1 and its inhibition by prosurvival Bcl-2 family proteins. Autophagy 4: 519-520, 2008.

11. Maiuri MC, Criollo A, Tasdemir E, et al: $\mathrm{BH} 3$-only proteins and $\mathrm{BH} 3$ mimetics induce autophagy by competitively disrupting the interaction between Beclin 1 and $\mathrm{Bcl}-2 / \mathrm{Bcl}-\mathrm{X}(\mathrm{L})$. Autophagy 3: 374-376, 2007.

12. Zalckvar E, Berissi H, Eisenstein M and Kimchi A: Phosphorylation of Beclin 1 by DAP-kinase promotes autophagy by weakening its interactions with Bcl-2 and Bcl-XL. Autophagy 5: 720-722, 2009.
13. Anorlu RI: What is the significance of the HPV epidemic? Can $\mathrm{J}$ Urol 15: 3860-3865, 2008.

14. Weinstein LC, Buchanan EM, Hillson C and Chambers CV: Screening and prevention: cervical cancer. Prim Care 36: 559-574, 2009.

15. Singh KC, Agarwal A, Agarwal S, et al: 'Quick course' neoadjuvant chemotherapy with cisplatin, bleomycin and vincristine in advanced cervical cancer. Gynecol Obstet Invest 58: 109-113, 2004.

16. van Luijk IF, Coens C, van der Burg ME, et al: Phase II study of bleomycin, vindesine, mitomycin $\mathrm{C}$ and cisplatin (BEMP) in recurrent or disseminated squamous cell carcinoma of the uterine cervix. Ann Oncol 18: 275-281, 2007.

17. Benson C, White J, De Bono J, et al: A phase I trial of the selective oral cyclin-dependent kinase inhibitor seliciclib (CYC202; R-roscovitine), administered twice daily for 7 days every 21 days. Br J Cancer 96: 29-37, 2007.

18. Sherr CJ: Cancer cell cycles. Science 274: 1672-1677, 1996.

19. Guzi T: CYC-202 Cyclacel. Curr Opin Investig Drugs 5: 1311-1318, 2004.

20. Coley HM, Shotton CF and Thomas H: Seliciclib (CYC202; r-roscovitine) in combination with cytotoxic agents in human uterine sarcoma cell lines. Anticancer Res 27: 273-278, 2007.

21. Dey A, Wong ET, Cheok CF, Tergaonkar V and Lane DP: R-roscovitine simultaneously targets both the 53 and NF-kappaB pathways and causes potentiation of apoptosis: implications in cancer therapy. Cell Death Differ 15: 263-273, 2008.

22. Duffin R, Leitch AE, Sheldrake TA, et al: The CDK inhibitor, R-roscovitine, promotes eosinophil apoptosis by down-regulation of Mcl-1. FEBS Lett 583: 2540-2546, 2009.

23. Goodyear S and Sharma MC: roscovitine regulates invasive breast cancer cell (MDA-MB231) proliferation and survival through cell cycle regulatory protein cdk5. Exp Mol Pathol 82: 25-32, 2007.

24. Maurer M, Komina O and Wesierska-Gadek J: roscovitine differentially affects asynchronously growing and synchronized human MCF-7 breast cancer cells. Ann NY Acad Sci 1171: 250-256, 2009

25. Mitchell C, Park MA, Zhang G, et al: Extrinsic pathway- and cathepsin-dependent induction of mitochondrial dysfunction are essential for synergistic flavopiridol and vorinostat lethality in breast cancer cells. Mol Cancer Ther 6: 3101-3112, 2007.

26. Nair BC, Vallabhaneni S, Tekmal RR and Vadlamudi RK: roscovitine confers tumor suppressive effect on therapy-resistant breast tumor cells. Breast Cancer Res 13: R80, 2011.

27. Ribas J, Boix J and Meijer L: (R)-roscovitine (CYC202, Seliciclib) sensitizes SH-SY5Y neuroblastoma cells to nutlin-3-induced apoptosis. Exp Cell Res 312: 2394-2400, 2006.

28. Zolnierczyk JD, Bloński JZ, Robak T, Kiliańska ZM and Wesierska-Gadek J: roscovitine triggers apoptosis in B-cell chronic lymphocytic leukemia cells with similar efficiency as combinations of conventional purine analogs with cyclophosphamide. Ann NY Acad Sci 1171: 124-131, 2009.

29. Wesierska-Gadek J, Gueorguieva M and Horky M: roscovitine-induced up-regulation of p53AIP1 protein precedes the onset of apoptosis in human MCF-7 breast cancer cells. Mol Cancer Ther 4: 113-124, 2005.

30. Lambert LA, Qiao N, Hunt KK, et al: Autophagy: a novel mechanism of synergistic cytotoxicity between doxorubicin and roscovitine in a sarcoma model. Cancer Res 68: 7966-7974, 2008.

31. Houel-Renault L, Philippe L, Piquemal M and Ciapa B: Autophagy is used as a survival program in unfertilized sea urchin eggs that are destined to die by apoptosis after inactivation of MAPK1/3 (ERK2/1). Autophagy 9: 1527-1539, 2013.

32. Sinha $S$ and Levine B: The autophagy effector Beclin 1: a novel BH3-only protein. Oncogene 27 (Suppl 1): S137-S148, 2008.

33. Wei Y, Sinha S and Levine B: Dual role of JNK1-mediated phosphorylation of Bcl-2 in autophagy and apoptosis regulation. Autophagy 4: 949-951, 2008.

34. Zalckvar E, Berissi H, Mizrachy L, et al: DAP-kinase-mediated phosphorylation on the $\mathrm{BH} 3$ domain of beclin 1 promotes dissociation of beclin 1 from Bcl-XL and induction of autophagy. EMBO Rep 10: 285-292, 2009.

35. Kabeya Y, Mizushima N, Ueno T, et al: LC3, a mammalian homologue of yeast Apg8p, is localized in autophagosome membranes after processing. Embo J 19: 5720-5728, 2000. 